\title{
Estimation of Surface Crack Depth using Rayleigh Waves by Electromagnetic Acoustic Transducers
}

\author{
Cunfu He, Peng Deng, Yan Lu, Xiucheng Liu, Zenghua Liu, Jingpin Jiao and Bin Wu \\ College of Mechanical Engineering and Applied Electronics Technology, Beijing University of Technology, Beijing \\ 100124, China.
}

(Received 23 February 2017; accepted 31 May 2017)

\begin{abstract}
A quantitative characterization method is introduced for estimating surface crack depth using Rayleigh waves in pitch-catch mode by electromagnetic acoustic transducers (EMATs). The method employs the experimentally determined reflection and transmission coefficients of Rayleigh waves scattered at a surface crack, which will be compared to the reference curves obtained from two-dimensional finite element method (FEM) simulations based on variable crack depth. Three EMAT couples with different centre frequencies were employed to extend the measuring range, and to implement the quantitative characterization of crack depth. The reference curves and measurement results were verified to be repeatable with great accuracy, which shows a maximum error of $17 \%$ with crack depth ranging from $0.2-3.0 \mathrm{~mm}$.
\end{abstract}

\section{INTRODUCTION}

Surface cracks generated by surface strains in structurally critical materials, such as rails, steel plates, thick-walled pipes, etc., can greatly affect the structural integrity, either during manufacturing process or in the service period. Nondestructive testing (NDT) is therefore becoming an increasingly essential tool for ensuring the structural integrity and safety. ${ }^{1}$ Although to confirm the presence and location of cracks by NDT is of great importance, an accurate estimation of crack depth is much more crucial for structural health evaluation. $^{2}$

Rayleigh waves are extensively applied in NDT for the detection of surface defects, and are particularly appropriate for crack depth sizing. ${ }^{3}$ Rayleigh wave propagates near the surface with its velocity independent of frequency, ${ }^{4}$ and attenuates exponentially when travelling from the surface. ${ }^{5}$ The energy of Rayleigh waves is concentrated near the surface, making them more sensitive to surface cracks compared with bulk waves. ${ }^{6}$ Rayleigh waves can also propagate along curved surfaces, such as pipes with fairly large diameters, in which variation of velocity and phase is negligible. ${ }^{4}$ Furthermore, Rayleigh waves can be employed for the characterization of angled surface cracks. ${ }^{1,7}$

Crack depth can be estimated by the scattering phenomena when Rayleigh waves meet a crack. The travelling time and amplitude information of the incident, reflected, and transmitted Rayleigh waves carry the detail characteristics of the crack. $^{3,8}$ In time domain, the propagating time of a surface wave along the crack's cliffs can be measured to estimate the extent of crack penetration..$^{9,10}$ It is an effective approach for depth characterization when the depth is greater than $0.8 \lambda$, in which the $\lambda$ is the corresponding wavelength. Date ${ }^{8}$ employed the surface wave transmission method to measure the crack depth to a scale as small as $0.82 \mathrm{~mm}$ with a mean error of $0.01 \mathrm{~mm}$ using a $5 \mathrm{MHz}$ probe. Similarly, Baby ${ }^{11}$ employed time-of-flight diffraction to estimate crack depth in the range of 1.68-19.04 mm with excellent accuracy by using $4 \mathrm{MHz}$ angle beam probes. However, time-domain methods are not appropriate for the evaluation of a surface crack with a depth less than $0.8 \lambda$, due to the irregular time delay effect. ${ }^{3}$ Hence, the relative amplitudes of the incident, reflected, and transmitted Rayleigh waves at a crack, in form of reflection and transmission coefficients, become prospective for crack depth evaluation. Kino ${ }^{12}$ and Ault ${ }^{13}$ adopted the reciprocity theory to associate the scattering coefficients with the characteristics of the flaw, and Tien ${ }^{14}$ extended this formulation for Rayleigh waves to observe the surface cracks in ceramics. Reflection and transmission coefficient curves can be obtained by numerical calculation and simulation. ${ }^{15-17}$ In contrast with time-domain methods, the methods based on reflection and transmission coefficients are more efficient for estimation of miniscule surface cracks. Resch ${ }^{18}$ utilized the reflection coefficient of Rayleigh waves to estimate the crack depth less than $0.05 \mathrm{~mm}$ with an average error of $9.6 \%$ using surface acoustic wave wedge transducers centred around 3.5 MHz. Hevin ${ }^{19}$ detected cracks in concrete structures experimentally, and determined the crack depth within an error of $15 \%$ of the actual size. However, when the crack depth is greater than $0.3 \lambda$, both the reflection and transmission coefficients oscillate fiercely as crack depth increases, resulting in an ambiguous relationship between the scattering coefficients and the crack depth. ${ }^{20}$ It is therefore difficult to accurately estimate crack depth by reflection and transmission coefficients of Rayleigh waves with a single centre frequency. ${ }^{21}$

In previous work, piezoelectric transducers and lasers have been widely used to generate and detect Rayleigh waves. ${ }^{22-25}$ Piezoelectric transducers are efficient for energy transformation, but the quality of received signal is critically affected by coupling conditions. Generally, laser ultrasonic equipment is much more expensive and complicated for practical applications. Edwards ${ }^{2}$ and $\mathrm{Jian}^{21}$ employed electromagnetic acoustic transducers (EMATs) to generate low frequency wideband Rayleigh waves for estimation of surface crack depth. EMATs are non-contact ultrasonic transducers that do not require a couplant, and appear to be much more robust despite the coupling conditions than piezoelectric transducers. ${ }^{26}$ Meanwhile, the implementation of EMATs shows great simplicity and repeatability, compared with laser ultrasonics. ${ }^{27}$ 


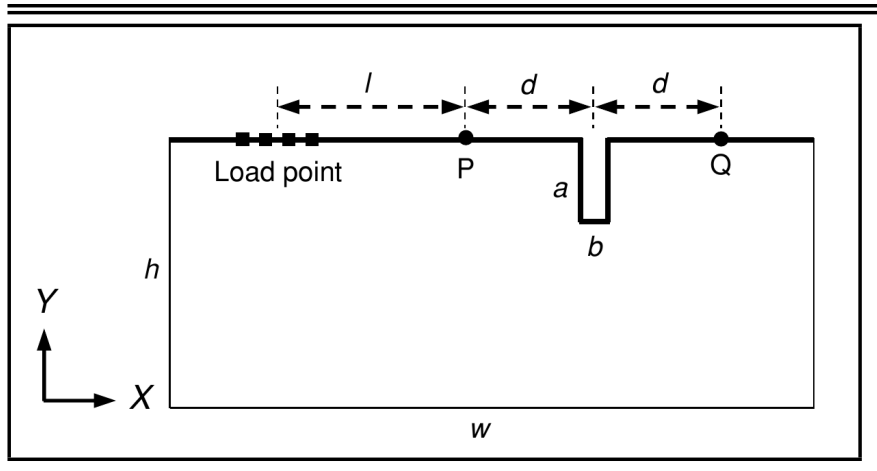

Figure 1. Two-dimensional geometrical model for FEM calculation of Rayleigh wave reflection and transmission coefficients.

In this research, Rayleigh waves based on EMATs will be adopted to estimate the depth of surface cracks, using reference reflection and transmission coefficient curves and a measurement procedure. The reference curves are established by two-dimensional (2D) finite element method (FEM) simulation with variable crack depth, and the measurement procedure is employed to compensate the amplitude decay of the received signals related to the propagation distance; both of them are verified with excellent accuracy.

Three EMAT couples with different centre frequencies of $0.5,1.0$, and $1.5 \mathrm{MHz}$ are fabricated to estimate the crack depth in order to extend the measuring range. The EMATs simply consist of a magnet and meander-line coil. When they are stacked up on the steel plate and drove by alternating current, Rayleigh wave will be generated through the Lorentz force, magnetostriction force, and magnetization force.

Multifrequency method and multiparameter are used to estimate the crack depth, by which the measurement accuracy is improved. Indeed, for a certain crack, it is detected by Rayleigh waves with different centre frequencies, and its depth is predicted by combining the measurements of different frequencies according to the corresponding reference curves. Finally, this proposed method demonstrates a maximum error of $17 \%$ with crack depth ranging from $0.2-3.0 \mathrm{~mm}$.

\section{SCATTERING COEFFICIENTS CALCULATED BY FEM SIMULASION}

\subsection{FEM Model}

A 2-D FEM model is established to obtain the simulated reflection and transmission coefficients of Rayleigh waves at a series of ideal cracks with varying depth, using COMSOL Multiphysics (COMSOL Inc., Sweden). As illustrated in Fig. 1, a steel plate of thickness $h$ and width $w$ is considered with a rectangular groove of depth a and a constant width $b=0.5 \mathrm{~mm}$, which is perpendicular to the plate surface.

The Cartesian coordinate system originates from the lower left corner of the steel plate, and $h$ is greater than $6 \lambda$ in order to ensure the surface propagation. The value of $a$ varies from $0.1 \mathrm{~mm}$ to $2 \lambda$. The top surface of the steel plate and the crack are set to free boundaries, as shown in bold line in Fig. 1, while the others set to low-reflecting boundaries in order to simplify the received signal.

Rayleigh waves with different exciting frequencies of 0.5 , 1.0 , and $1.5 \mathrm{MHz}$, with corresponding wavelengths $\lambda$ of $6 \mathrm{~mm}$, $3 \mathrm{~mm}, 2 \mathrm{~mm}$, and propagating velocity of $3000 \mathrm{~m} / \mathrm{s}$ are employed in the FEM calculations. The mesh grid in the model (a)

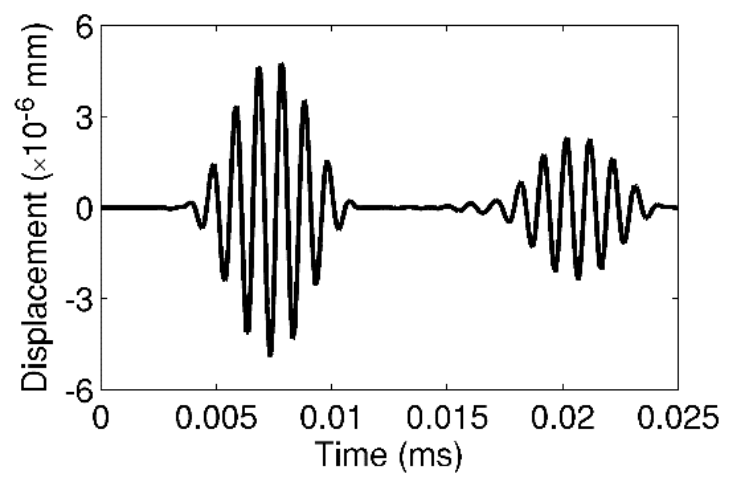

(b)

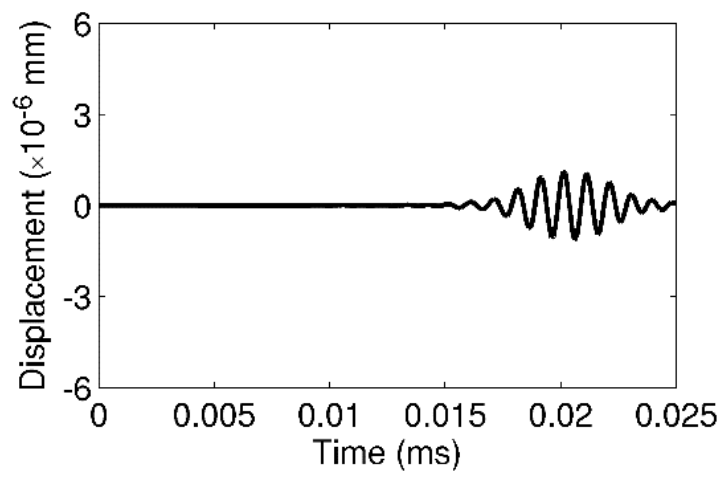

Figure 2. The out-of-plane displacements at (a) point $P$ and (b) point $Q$ with a center frequency of $1.0 \mathrm{MHz}$, and depth $a=1.0 \mathrm{~mm}$.

consists of free triangular elements, and the size of the elements is less than $0.1 \lambda$. A five-cycle force loading modulated by a Hanning window is applied perpendicularly to the top surface, located at a distance $l$ from the first detection point. Four loading points are used, in which the interval of these points ought to be $\lambda$. It should be noticed that, the $l$ and the distance $d$ are set to be sufficiently large to avoid the phenomena of wave overlapping.

\subsection{FEM Results}

The out-of-plane displacements at detection point $P$ and $Q$ are obtained by FEM calculations for each Rayleigh wave centre frequency. Figure 2 shows the out-of-plane displacements at (a) point $P$ and (b) point $Q$ for a centre frequency of $1.0 \mathrm{MHz}$ and $a=1.0 \mathrm{~mm}$. Figure 2(a) shows the outof-plane displacements of the Rayleigh wave coming from the load point directly and the Rayleigh wave reflected from the groove. Figure 2(b) shows the out-of-plane displacements of the Rayleigh wave, which is transmitted through the groove.

In the following context, the reflection and transmission coefficients are defined as the ratio of the amplitude of reflected and transmitted Rayleigh waves to the incident ones, respectively. Figure 3 presents the calculated reflection and transmission coefficients of Rayleigh waves in different centre frequencies with respect to the ratio $a / \lambda$. In Figs. 3(a) and (b), these three curves with different excitation frequencies exist slight discrepancy. This is due to the different ratio of $b / \lambda{ }^{3}$ in which $\mathrm{b}$ is a given width and $\lambda$ is a variable corresponding to frequency. Additionally, the value of $b / \lambda$ induces a greater impact on both reflection and transmission coefficients when $a / \lambda<0.5$. 


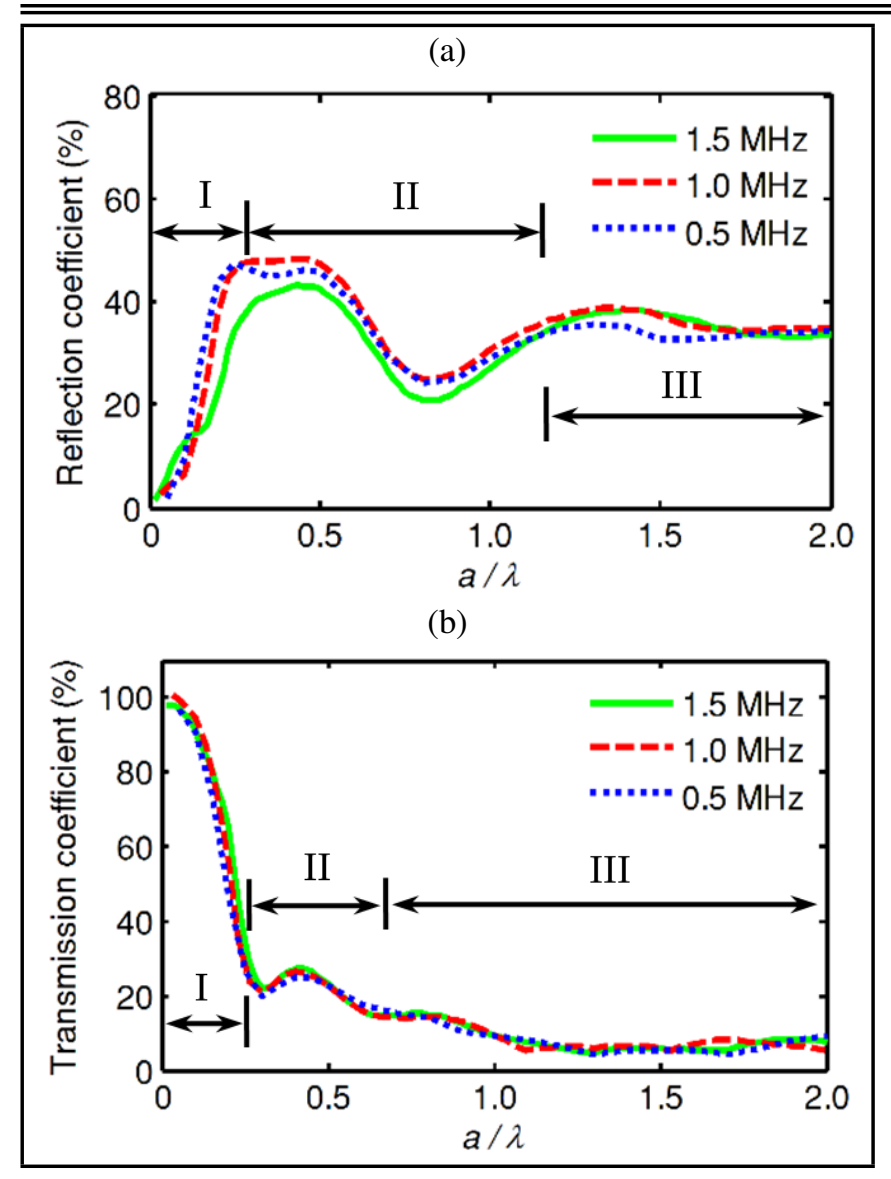

Figure 3. Calculated (a) reflection and (b) transmission coefficient curves at surface grooves of varying depth a and given width $b=0.5 \mathrm{~mm}$ with center frequencies of $1.5 \mathrm{MHz}, 1.0 \mathrm{MHz}$ and $0.5 \mathrm{MHz}$.

Clearly, it is should be noticed that the reflection and transmission coefficient curves calculated from different frequencies are not monotonic with respect to $a / \lambda$, as shown in Fig. 3. The reflection coefficient curves increase proportionally when $\mathrm{a} / \lambda<0.3$, at the same time the transmission coefficient curves decrease. When $a / \lambda>0.3$, both reflection and transmission coefficient curves oscillate, and then they vary slowly. Hence the coefficient curves are divided into monotonic region (region I), oscillating region (region II), and slowly varying region (region III), as exhibited in Fig. 3. The reflection and transmission coefficient curves obtained from the FEM results clearly indicate that Rayleigh waves are sensitive to very shallow cracks. Within the range of $a / \lambda<0.3$, the crack depth can be readily estimated based on the reflection and transmission coefficients. However, if the whole range of the curve is taken into consideration, and one single reflection coefficient is given, it will lead to the result of ambiguous crack depth. That is to say, it is difficult to estimate the crack depth accurately using single frequency and single scattering coefficient.

To sum up, all the reflection and transmission coefficient curves are function of $a / \lambda$, rather than $a$ alone. If a reflection and/or transmission coefficient is measured for an unknown crack using single frequency, there may be more than one corresponding crack depth. Then, it suggests that additional frequency-dependent information may be useful for accurate estimation of crack depth. As such, for a given crack, the ratio $a / \lambda$ varies according to the Rayleigh wavelength, and the reflection and transmission coefficients of Rayleigh waves with different centre frequencies may present distinct differ-

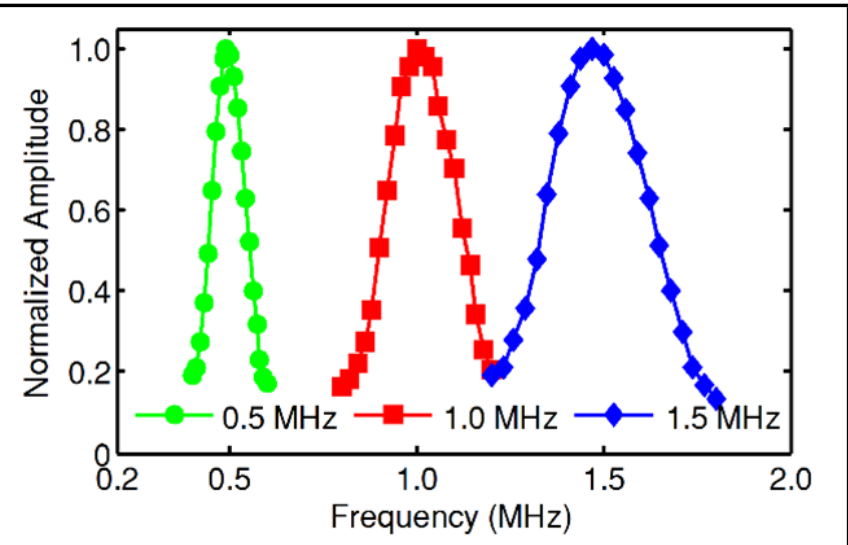

Figure 4. Frequency characteristics of the EMATs with different center frequencies.

ences associated with their different locations along the reflection and transmission coefficient curves, by which the crack depth might be easily estimated.

\section{EXPERIMENTAL ARRANGEMENT}

\subsection{Specimens}

Two standard specimens are used in the experimental study. Both are $25 \mathrm{~mm}$ thick steel plates (45\#), and specimen 1 is $450 \mathrm{~mm} \times 600 \mathrm{~mm}$ of its width $\times$ length, while specimen 2 is $400 \mathrm{~mm} \times 500 \mathrm{~mm}$. Five grooves with depths of $0.5-2.5$ $\mathrm{mm}$ in $0.5 \mathrm{~mm}$ interval are machined as the crack on specimen 1, and eight grooves with depths of $0.2,0.3,0.4,0.6,0.7,0.8$, 0.9 , and $3.0 \mathrm{~mm}$ are machined on specimen 2 . The groove depth is denoted as $a_{0}$. The lengths of all grooves are $30 \mathrm{~mm}$ and the widths are $0.5 \mathrm{~mm}$, which is also used in the FEM models. All the grooves are separated from each other with a distance of $90 \mathrm{~mm}$. Measurements of the grooves verify that a dimensional tolerance of $50 \mu \mathrm{m}$ can be satisfied.

\subsection{Surface Wave EMATs}

The excellent consistency and repeatability exhibited by EMATs are crucial for the accurate estimation of crack depth. Three surface wave EMAT couples were fabricated to generate and detect Rayleigh waves. Every EMAT employs a meanderline coil of six turns, in which the length of the wire in the coil is $20 \mathrm{~mm}$. The intervals between adjacent lines for each EMAT couple are $3.0,1.5$, and $1.0 \mathrm{~mm}$, respectively, which provide Rayleigh wavelengths of $6.0,3.0$, and $2.0 \mathrm{~mm}$, corresponding to centre frequencies of $0.5,1.0$, and $1.5 \mathrm{MHz}$, respectively. The coils are $56 \mathrm{~mm}$ long and $30 \mathrm{~mm}$ wide. The permanent magnet used is NdFeB35 with size of $50 \times 30 \times 10 \mathrm{~mm}^{3}$. The practical frequency performance was evaluated, and the results demonstrated good agreement with design specifications. The $-6 \mathrm{~dB}$ bandwidth of the three EMAT couples were about $22 \%$, as Fig. 4.

\subsection{Experimental Setup}

The experimental setup comprised of a RPR-4000 Computer Controlled High Power Pulser/Receiver (RITEC Inc., USA), impedance analyser (Agilent Technologies, USA), digital oscilloscope (Tektronix Inc., USA), steel specimen, transmitting, and receiving surface wave EMATs (denoted as transmitter and 


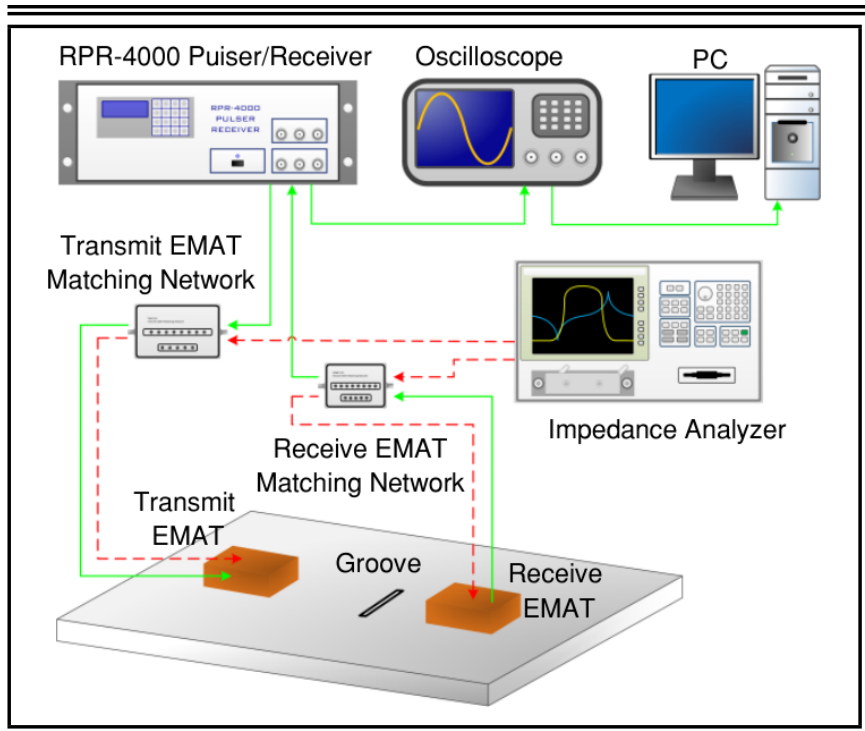

Figure 5. Rayleigh wave generation and detection using surface wave EMATs on a steel specimen. The EMATs were matched using an impedance analyzer.

receiver, respectively), and their electrical matching networks, as shown in Fig. 5. Pitch-catch mode was adopted to measure the incident, reflected, and transmitted Rayleigh waves (denoted as I-Wave, R-Wave and T-Wave). Further, EMAT matching networks and an impedance analyser was used to implement the impedance matching, so as to improve the efficiency of energy transfer. The green line was the data/control flow, while the red dash line represented the schematic of impedance matching. A 6-cycle sinusoidal tone burst modulated by a rectangular window was utilized to drive the transmitter to project Rayleigh waves into the specimen. Centre frequencies of 0.5 , 1.0 , and $1.5 \mathrm{MHz}$ were used, corresponding to the three different EMAT couples. The transmitter and receiver were positioned perpendicular to the groove, and aligned along with their centre lines. The transmitter was $L_{1}$ distance away from the groove centre. The I-Wave and R-Wave were detected with the receiver placed on the same side of the groove as the transmitter, as shown in Fig. 6(a). For detection of the T-Wave, the receiver was placed a distance $L_{2}$ from the groove on the other side, as shown in Fig. 6(b). The received voltage signal would be filtered by a band pass filter $(0.4-2.5 \mathrm{MHz}$ for $0.5 \mathrm{MHz}$ transducer, $0.8-2.5 \mathrm{MHz}$ for $1.0 \mathrm{MHz}$ and $1.5 \mathrm{MHz}$ transducers), and averaged 256 times in oscilloscope.

\subsection{Measurement Procedures}

The reflection and transmission coefficients were obtained from the 2-D FEM simulations, respectively, as the ratios of reflected and transmitted amplitudes to those of incident surface wave, and no attenuation was taken into account during wave propagation. However, in experiments, the amplitudes of Rayleigh waves are attenuated along with the propagating distance due to diffusion of the acoustic beam and the material absorption. Clearly, the experimental reflection and transmission coefficients would be considerably smaller than the values obtained in the absence of attenuation. Hence, the practical attenuation should be taken into consideration, and the experimental reflection and transmission coefficients should be compensated and obtained as follows:

i. the attenuation curves of Rayleigh waves propagation ought to be experimentally measured first using the EMAT

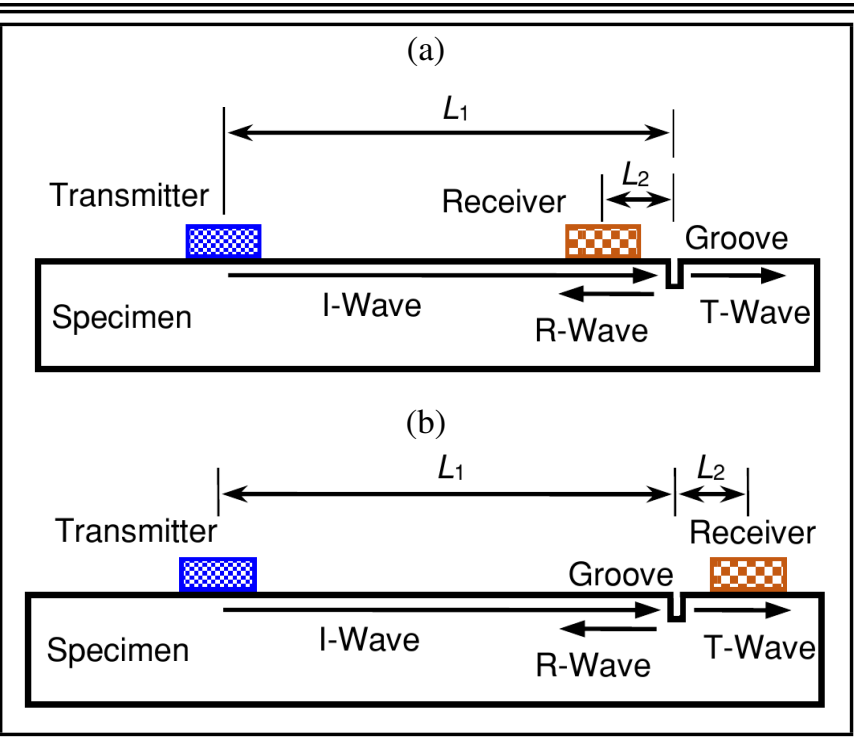

Figure 6. The arrangement of transmitter and receiver in experiments for the detection of (a) I-Wave and R-Wave, and (b) T-Wave at a groove on the steel specimen.

couples;

ii. the incident, reflected, and transmitted surface waves were detected using the receive EMATs as outlined in section 3.3;

iii. the amplitudes of incident, reflected, and transmitted waves were compensated according to the attenuation curves and their own propagation distances;

iv. the reflection and transmission coefficients were calculated using the compensated amplitudes, denoted as $C_{r e f}$ and $C_{\text {tran }}$, and then these coefficients can be compared with the FEM results directly.

It should be noticed that the attenuation curves of Rayleigh waves propagating in different specimens may vary due to different surface conditions, such as surface texture and roughness. Therefore, the attenuation curves should be experimentally obtained for every single specimen.

In the experiments, Rayleigh waves were generated by the transmitter located relatively far away from the groove to eliminate disturbances owing to the generated body waves. The receiver was placed near the groove to improve their detection capabilities. The arrangements of the two EAMTs in the experiments are presented in Fig. 6. The transmitter was fixed at a constant distance $L_{1}=335 \mathrm{~mm}$ from the centre of the groove. The receiver was arranged on either side of the groove at a distance $L_{2}=30 \mathrm{~mm}$ to detect reflected or transmitted waves.

\section{RESULTS AND DISCUSSION}

A measurement result for $a=1.0 \mathrm{~mm}$ is illustrated in Fig. 7 with a centre frequency $1.0 \mathrm{MHz}$. The results given in Figs. 7(a) and (b) correspond to the measurement setups illustrated in Figs. 6(a) and (b), respectively. Wave package (WP) 1 shown in Fig. 7(a) with a minimal arrival time $t_{1}=0.122 \mathrm{~ms}$ is Rayleigh wave, which directly propagating from transmitter to receiver is adopted as I-Wave. The I-Wave continued to propagate forward, and was reflected and transmitted at the groove. 


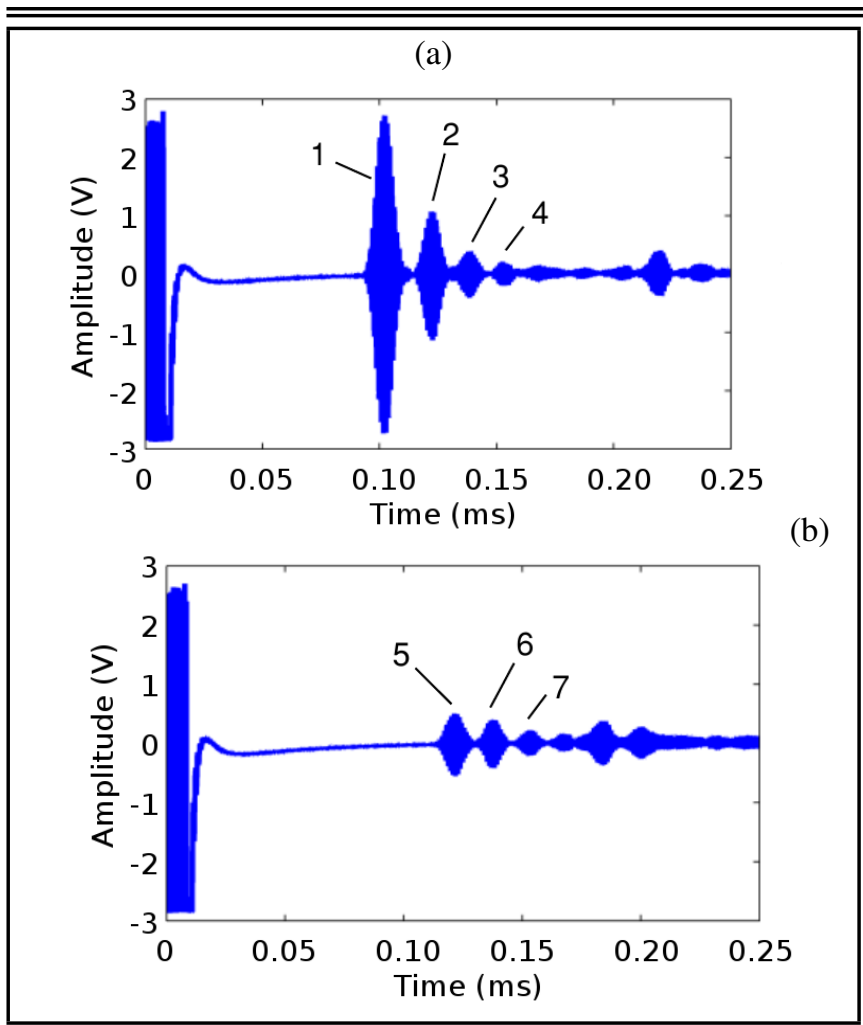

Figure 7. Average signals based on summations of over 256 individual six cycle excitations detected by the receiver with a center frequency of $1.0 \mathrm{MHz}$ and a crack depth $a=1.0 \mathrm{~mm}$ : (a) I-Wave and R-Wave; (b) T-Wave.

The R-Wave and T-Wave would be detected by receiver at the left and right side of the groove. The R-Wave is presented as WP 2 in Fig. 7(a) and the T-Wave is presented as WP 5 in Fig. 7(b). The peak-to-peak amplitudes of WP 1, 2, and 5 were compensated, and then used to calculate $C_{\text {ref }}$ and $C_{\text {tran }}$.

The additional wave packages (WP 3, 4, 6, 7 in Fig. 7) are actually Rayleigh waves transformed at the groove edge from shear wave, which was previously scattered at the groove by IWave, and reflected from the lower boundary, ${ }^{28,29}$ as presented in Fig. 8. The WP 3 and 6 were generated by the first reflected shear wave, and the WP 4 and 7 were generated by the second reflected shear wave. The time differences between adjacent wave packages, denoted as $\Delta t_{2,3}, \Delta t_{3,4}, \Delta t_{5,6}$, and $\Delta t_{6,7}$ were identical. In addition, the amplitudes of WP 2-4 and WP 5-7 decreased gradually, as shown in Fig. 7.

\subsection{Experimental Verification of Reflection and Transmission Coefficients}

The reflection and transmission coefficient curves obtained by FEM simulations were verified by experimental measurements for various depth $a$. Measurements were conducted three times with three exciting centre frequencies for each groove on specimen 1. Figure 9 presents the comparisons of the reflection and transmission coefficients obtained from the FEM results (solid lines) and the three experimental results (denoted by open circles, squares and rhombuses) with centre frequencies of $0.5,1.0$, and $1.5 \mathrm{MHz}$, respectively, which are plotted with respect to a rather than $a / \lambda$ to illustrate how the centre frequency affects the location of Cref and Ctran on the overall reflection and transmission coefficient curves.

In the multi-frequency measurements, in Fig. 9, for a given depth $a$, the measured coefficients are located in different re- (a)

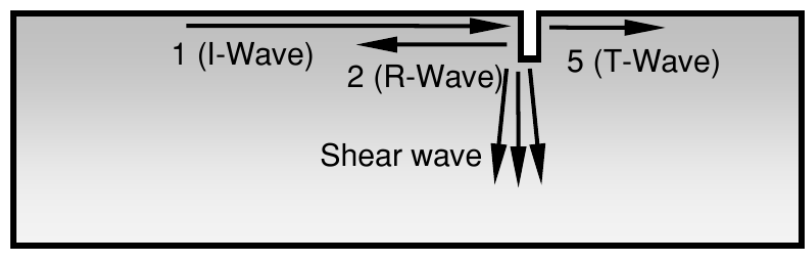

(b)

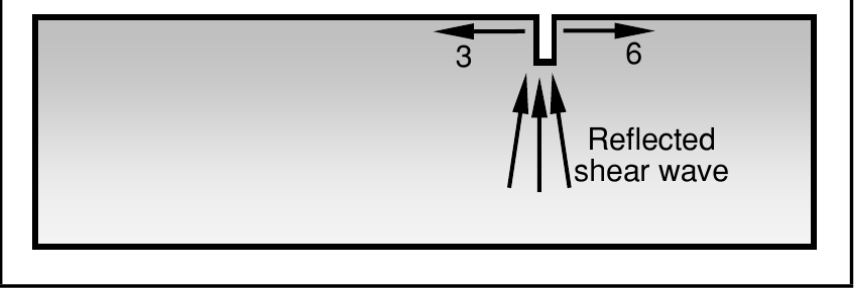

Figure 8. Wave propagation path in experiments.

Table 1. The experimental reflection and transmission coefficients (the green circles in Fig. 9) and the corresponding values of a for the groove with depth $a_{0}=2.0 \mathrm{~mm}$

\begin{tabular}{||c|c|c|c|c|}
\hline $\begin{array}{c}\text { Frequency } \\
(\mathrm{MHz})\end{array}$ & $\begin{array}{c}C_{\text {ref }} \\
(\%)\end{array}$ & $\begin{array}{c}C_{\operatorname{tran}} \\
(\%)\end{array}$ & $\begin{array}{c}\text { Corresponding } a \\
\text { of } C_{\text {ref }}(\mathrm{mm})\end{array}$ & $\begin{array}{c}\text { Corresponding } a \\
\text { of } C_{\text {tran }}(\mathrm{mm})\end{array}$ \\
\hline 0.5 & 42.13 & 22.78 & $2.287 / 3.041$ & $\begin{array}{c}1.754 / \\
2.003 / 3.036\end{array}$ \\
\hline 1.0 & 29.84 & 12.35 & $\begin{array}{c}0.539 / \\
2.115 / 2.955\end{array}$ & $\begin{array}{c}0.287 / \\
1.494 / 1.853\end{array}$ \\
\hline 1.5 & 26.05 & 8.5 & $2.097 / 3.823$ \\
\hline
\end{tabular}

gions of coefficient curves. For example, when $f=0.5 \mathrm{MHz}$, $a=1 \mathrm{~mm}, C_{\text {ref }}$ and $C_{\text {tran }}$ are more likely to be located in the monotonic region (i.e., $a / \lambda<0.3$ ). However, when $f=1.5 \mathrm{MHz}, a=1 \mathrm{~mm}, C_{r e f}$ and $C_{\text {tran }}$ are more possibly to be located in the oscillating region and slowly varying region. As such, different $C_{\text {ref }}$ and $C_{\text {tran }}$ can be obtained for a given groove using Rayleigh waves with different centre frequencies. Hence, the depth a can be estimated directly by comparing the $C_{\text {ref }}$ and $C_{\text {tran }}$.

The observed deviations between the measurement and FEM results may be caused due to three different reasons. The first is related to the Rayleigh wave attenuation curve used to compensate the amplitudes of I-Wave, R-Wave, and T-Wave. The measured attenuation curve will fundamentally influence the experimental results. The second factor is that the relative positions between the transmitter and receiver will induce errors in $C_{r e f}$ and $C_{t r a n}$. The final one is that the electromagnetic interference from the measurement system can impact the measured amplitudes of the surface waves. Therefore, averaging should be employed.

\subsection{Depth Measurement of Grooves}

Each groove of specimens 1 and 2 was measured by the three EMAT couples with three different centre frequencies, then three groups of $C_{r e f}$ and $C_{t r a n}$ were obtained. Comparing each group of $C_{\text {ref }}$ and $C_{\text {tran }}$ with the corresponding coefficient curves calculated from FEM simulations, several distinctly different values of $a$ may be obtained owing to the non-monotonicity of the curves. For example, one group of $C_{r e f}$ and $C_{t r a n}$ of Rayleigh waves with different centre frequencies for groove with depth $a_{0}=2.0 \mathrm{~mm}$ (green circles in Fig. 9) was shown in Table 1, and the corresponding values of 
(a)

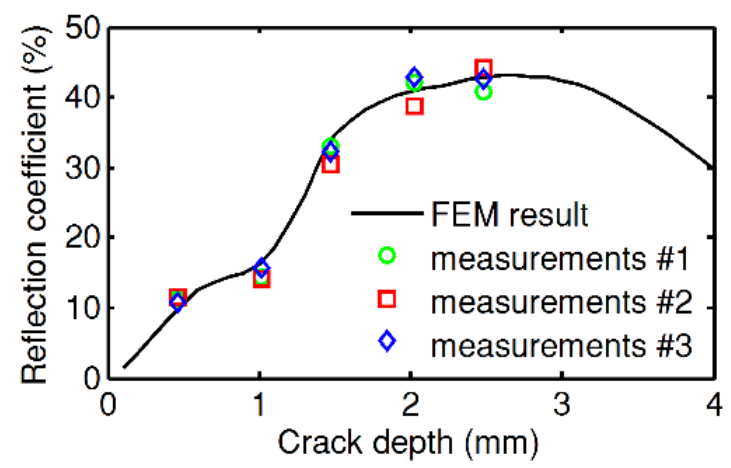

(c)

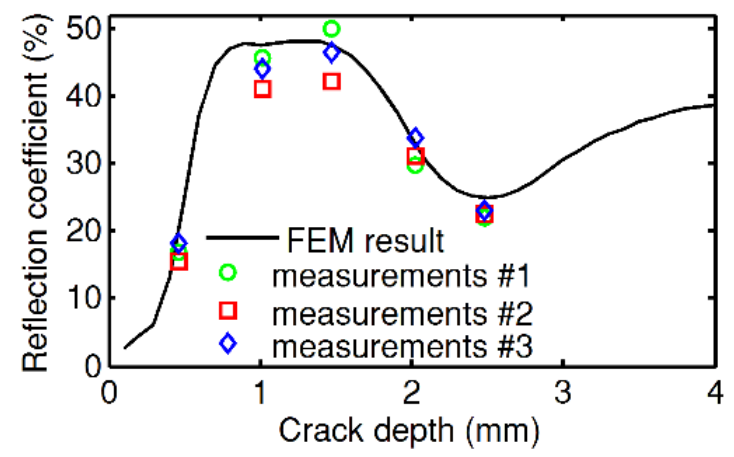

(e)

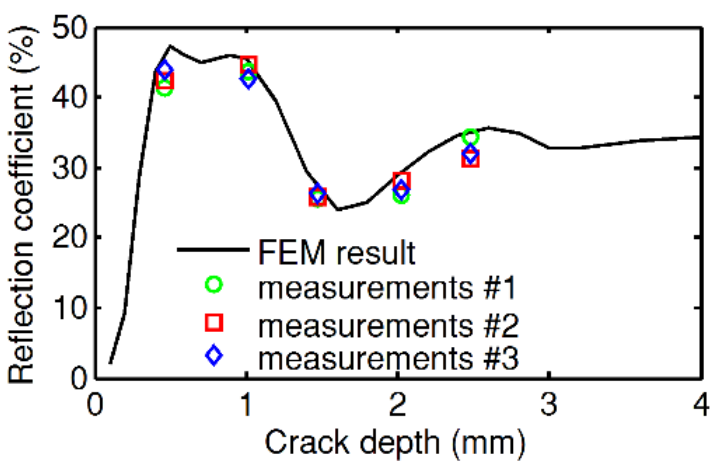

(b)

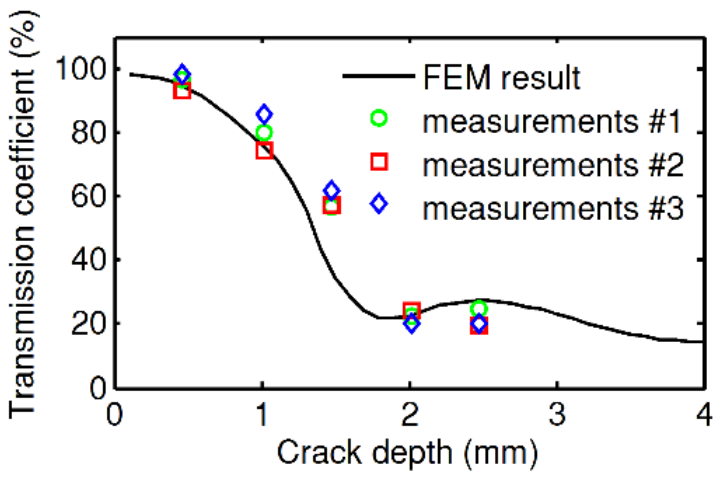

(d)

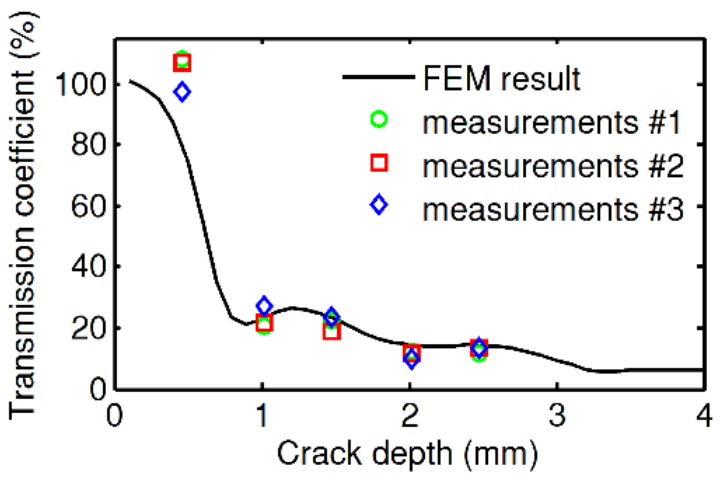

(f)

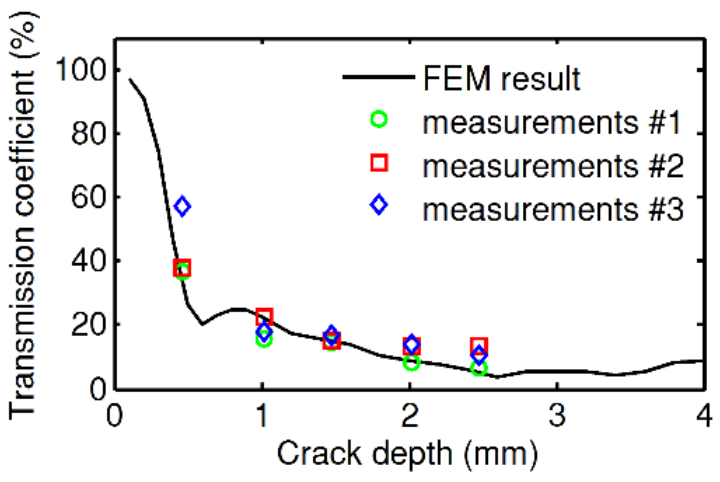

Figure 9. Reflection and transmission coefficients versus groove depth in the range $a_{0}=0-4$ mm: FEM result (solid line) and experimental measurements (open circles, squares and rhombuses).

$a$ compared with the reference curves were given as well. The gross errors of these values were eliminated; therefore, the remaining values were highly approximate to the actual depth value $a_{0}$. Then the average of the remaining values was calculated and considered as the estimate of groove depth $a_{\text {meas }}$.

Estimates of the depth for the 13 grooves were obtained by comprehensively analysing the reflection and transmission coefficients of Rayleigh waves with the three different centre frequencies, and the results are presented in Fig. 10 for values of $a=0.2-3.0 \mathrm{~mm}$. The evaluation for each groove was performed three times with equivalent experimental parameters, which are denoted by open circles, squares and rhombuses, respectively, in the figure. The measurement values $a_{\text {meas }}$ correlate well with the actual depth values $a_{0}$ over the entire detection range, and present good repeatability and accuracy.

A plot of the measurement accuracy with respect to $a_{0}$ is shown in Fig. 11. As can be observed, the accuracy decreased while $a_{0}<0.5 \mathrm{~mm}$. The lowest relative accuracy $17 \%$ showed up when $a 0=0.2 \mathrm{~mm}$, but it should be noted that the absolute error was only $33 \mu \mathrm{m}$. In the range $0.3 \mathrm{~mm} \leq a_{0} \leq 1.0 \mathrm{~mm}$, the relative accuracy is better than $10 \%$, while $1.5 \mathrm{~mm} \leq a_{0} \leq$ $3.0 \mathrm{~mm}$, the measurements deliver an excellent relative accuracy better than $5 \%$.

In the measurements, Rayleigh waves with different centre frequencies contribute different weights to the evaluation of ameas at different depth ranges owing to their different sensitivities. Rayleigh waves with the highest centre frequency of $1.5 \mathrm{MHz}$ provided a greater contribution to ameas for relatively shallow grooves such as those with depths of $0.2,0.3$, and $0.4 \mathrm{~mm}$, while Rayleigh waves with a centre frequency of $0.5 \mathrm{MHz}$ provided a greater contribution for grooves in the depth range of 1.5-3.0 mm. In fact, for $a 0=0.2 \mathrm{~mm}$, the reflected wave signal detected by the $0.5 \mathrm{MHz}$ Rayleigh wave was so weak as to be nearly submerged in the background noise.

It should be noticed that the values of $a_{\text {meas }}$ for $a 0=$ $2.5 \mathrm{~mm}$, while located in the relatively high measurement accuracy range of 1.5-3.0 (better than 5\%), expressed very evi- 


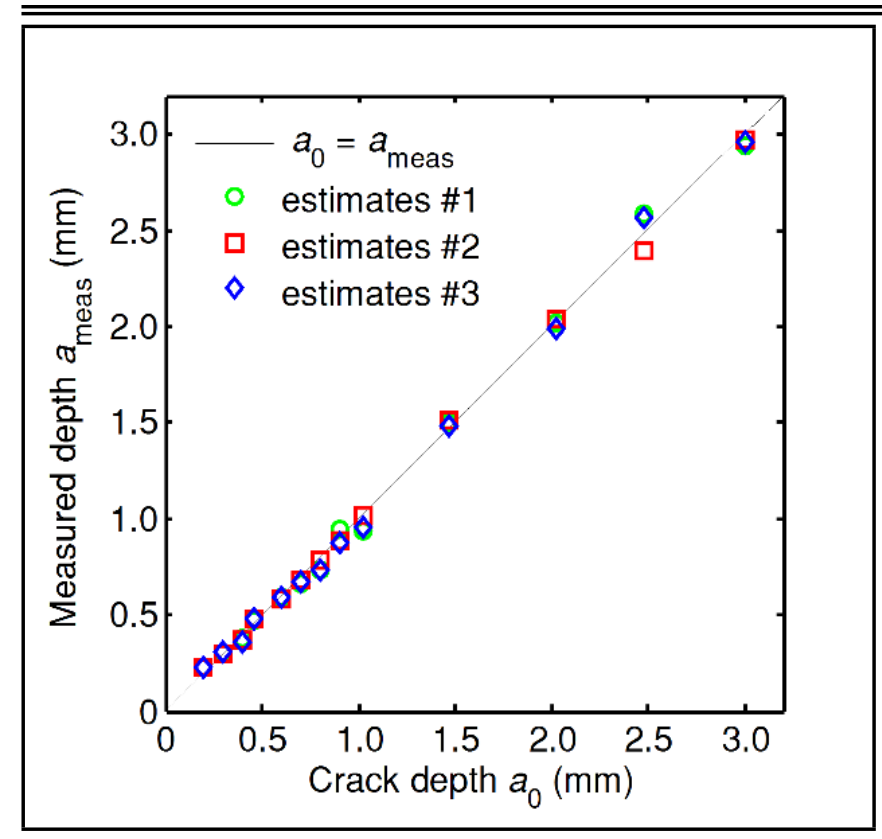

Figure 10. Estimations of groove depth $a_{\text {meas }}$ obtained under equivalent experimental conditions from the testing results of Rayleigh waves (open circles, squares and rhombuses) with respect to the actual depth $a_{0}$.

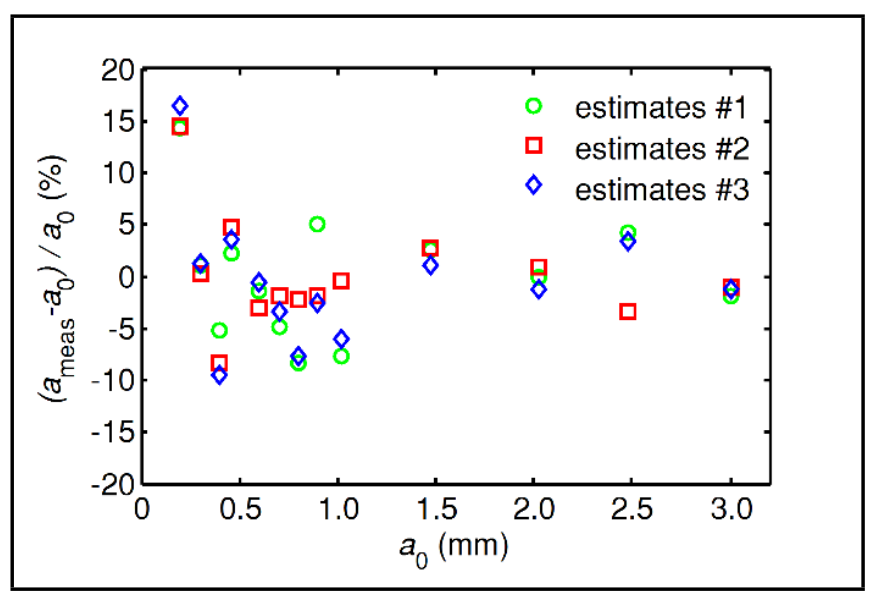

Figure 11. The measurement accuracy obtained under equivalent experimental conditions from the testing results of Rayleigh waves (open circles, squares and rhombuses).

dent dispersion around the value of $a_{0}$, as shown in Fig. 11. In this study, the measurements of the reflection and transmission coefficients for $a_{0}=2.5 \mathrm{~mm}$ are peculiar, in that a depth of 2.5 $\mathrm{mm}$ is located near local maxima in the reflection coefficient curves for centre frequencies of 0.5 and $1.5 \mathrm{MHz}$ (Figs. 7(a) and (e)), and near the local minimum in the reflection coefficient curve for a centre frequency of $1.0 \mathrm{MHz}$ (Fig. 7(c)). However, the slopes are relatively flat near extrema, such that the reflection coefficient is not sensitive to variations in the groove depth within this range. As such, the observed dispersion for $a_{0}=2.5 \mathrm{~mm}$ is not presently explainable.

A crack depth of $3.0 \mathrm{~mm}$ is probably the upper limit of the measurement range for the proposed approach employing centre frequencies of $0.5,1.0$ and $1.5 \mathrm{MHz}$. When the crack depth is greater than $3.0 \mathrm{~mm}$, the reflection coefficients of Rayleigh waves with 1.0 and $1.5 \mathrm{MHz}$ centre frequencies are nearly constant, and are therefore useless for estimating the crack depth. In addition, a crack depth of $0.2 \mathrm{~mm}$ is the lower limit for which the lowest frequency Rayleigh waves of $0.5 \mathrm{MHz}$ can detect reflected waves effectively. Despite these limitations, the detection range was extended. For a crack depth of $0.2 \mathrm{~mm}$, the value of $a / \lambda$ was as small as 0.1 for $\lambda=2 \mathrm{~mm}$ (i.e., $1.5 \mathrm{MHz}$ ).

\section{CONCLUSIONS}

A method using EMATs to transmit and receive Rayleigh waves in pitch-catch mode is developed for the depth characterization of small surface cracks, and the relative error of depth characterization ranges from $17 \%$ (with respect to crack depth of $0.2 \mathrm{~mm}$ ) to $1.8 \%$ (with respect to crack depth of $3 \mathrm{~mm})$.

The reflection and transmission coefficients are experimentally obtained and compensated by the measured attenuation curves. Then, the compensated coefficients are compared with the ones from FEM curves, while the depth of the grooves varies from $0.1 \mathrm{~mm}$ to $2 \lambda$ (which is related to the exciting frequency). Multi-frequency measurements (0.5, 1.0 and 1.5 MHz) are carried out for the depth characterization, in which the measurement range and reliability are improved significantly. This research on EMATs demonstrates a suitable approach to generate and detect Rayleigh waves.

The measured reflection and transmission coefficients are compensated according to each of their propagation distance, and the experimental results with compensation exhibit good agreement with the corresponding simulation curves. For a given depth, the measured coefficients from different excitation frequencies appear in different regions of the coefficient curve. This cross-referring operation plays a vital role in the characterization of crack depth, by which the depth can be finally extracted.

\section{ACKNOWLEDGEMENTS}

The research is supported by the National Natural Science Foundation of China (Grant No. 51235001, No. 11372016 , No. 51505013).

\section{REFERENCES}

1 Dutton, B., Clough, A.R., Rosli, M.H., Edwards, R.S. Non-contact ultrasonic detection of angled surface defects, NDT\&E International, 44 (4), 353-360, (2011). https://dx.doi.org/10.1016/j.ndteint.2011.02.001

2 Edwards, R.S., Dixon, S., Jian, X. Depth gauging of defects using low frequency wideband Rayleigh waves, Ultrasonics, 44 (1), 93-98, (2006). https://dx.doi.org/10.1016/j.ultras.2005.08.005

3 Masserey, B., Mazza, E. Ultrasonic sizing of short surface cracks, Ultrasonics, 46 (3), 195-204, (2007). https://dx.doi.org/10.1016/j.ultras.2007.02.001

${ }^{4}$ Cook, D.A., Berthelot, Y.H. Detection of small surfacebreaking fatigue cracks in steel using scattering of Rayleigh waves, NDT\&E International, 34 (7), 483-492, (2001). https://dx.doi.org/10.1016/s0963-8695(00)00080-3

5 Aggelis, D.G., Shiotani, T., Polyzos, D. Characterization of surface crack depth and repair evaluation using Rayleigh waves, Cement and 
Concrete Composites, 31 (1), 77-83, (2009). https://dx.doi.org/10.1016/j.cemconcomp.2008.09.008

6 Masserey, B., Mazza, E. Analysis of the near-field ultrasonic scattering at a surface crack, Journal of the Acoustical Society of America, 118 (6), 3585-3594, (2005). https://dx.doi.org/10.1121/1.2109407

7 Babich, V.M., Borovikov, V.A., Fradkin, L.J., Kamotski, V., Samokish, B.A. Scatter of the Rayleigh waves by tilted surface-breaking cracks, NDT\&E International, 37 (2), 105-109, (2004). https://dx.doi.org/10.1016/j.ndteint.2003.05.001

8 Date, K., Shimada, H., Ikenaga, N. Crack height measurement - an eveluation of the accuracy of ultrasonis timing methods, NDT International, 15 (6), 315-319, (1982). https://dx.doi.org/10.1016/0308-9126(82)90068-2

9 Silk, M.G. The determination of crack penetration using ultrasonic surface waves, NDT International, 9 (6), 290-297, (1976). https://dx.doi.org/10.1016/0308-9126(76)90071-7

10 Hudgell, R.J., Morgan, L.L., Lumb, R.F. Non-destructive measurement of the depth of surface-breaking cracks using ultrasonic Rayleigh waves, British Journal of NonDestructive Testing, 16 (5), 144-149, (1974).

11 Baby, S., Balasubramanian, T., Pardikar, R.J., Palaniappan, M., Subbaratnam, R. Time-of-flight diffraction (TOFD) technique for accurate sizing of surfacebreaking cracks, Insight, 45 (6), 426-430, (2003). https://dx.doi.org/10.1784/insi.45.6.426.52885

12 Kino, G.S. The application of reciprocity theory to scattering of acoustic waves by flaws, Journal of Applied Physics, 49 (6), 3190-3199, (1978). https://dx.doi.org/10.1063/1.325312

13 Auld, B.A. General electromechanical reciprocity relations applied to the calculation of elastic wave scattering coefficients, Wave Motion, 1, 3-10, (1979). https://dx.doi.org/10.1016/0165-2125(79)90020-9

14 Tien, J.J.W., Khuri-Yakub, B.T., Kino, G.S., Marshall, D.B., Evans, A.G. Surface acoustic wave measurements of surface cracks in ceramics, Journal of Nondestructive Evaluation, 2 (3-4), 219-229, (1981). https://dx.doi.org/10.1007/bf00570734

15 Achenbach, J.D., Gautesen, A.K., Mendelsohn, D.A. Ray analysis of surface-wave interaction with an edge crack, IEEE Transactions on Sonics And Ultrasonics, 27 (3), 124129, (1980). https://dx.doi.org/10.1109/t-su.1980.31161

16 Mendelsohn, D.A., Achenbach, J.D., Keer, L.M. Scattering of elastic waves by a surface-breaking crack, Wave Motion, 2 (3), 277-292, (1980). https://dx.doi.org/10.1016/01652125(80)90008-6

17 Angel, Y.C., Achenbach, J.D. Reflection and transmission of obliquely incident Rayleigh waves by a surface-breaking crack, Journal of the Acoustical Society of America, 75 (2), 313-319, (1984). https://dx.doi.org/10.1121/1.390473
18 Resch, M.T., Nelson, D.V., Yuce, H.H., Ramusat, G.F. A surface acoustic wave technique for monitoring the growth behavior of small surface fatigue cracks, Journal of Nondestructive Evaluation, 5 (1), 1-7, (1985). https://dx.doi.org/10.1007/bf00568757

19 Hevin, G., Abraham, O., Pedersen, H.A., Campillo, M. Characterization of surface cracks with Rayleigh waves: A numerical model, NDT\&E International, 31 (4), 289-297, (1998). https://dx.doi.org/10.1016/s0963-8695(98)80013-3

20 Jian, X., Dixon, S., Guo, N., Edwards, R. Rayleigh wave interaction with surface-breaking cracks, Journal of Applied Physics, 101 (6), 064906, (2007). https://dx.doi.org/10.1063/1.2435803

21 Jian, X., Dixon, S., Guo, N., Edwards, R.S., Potter, M. Pulsed Rayleigh wave scattered at a surface crack, Ultrasonics, 44, e1131-e1134, (2006). https://dx.doi.org/10.1016/j.ultras.2006.05.153

22 Masserey, B., Aebi, L., Mazza, E. Ultrasonic surface crack characterization on complex geometries using surface waves, Ultrasonics, 44, e957-e961, (2006). https://dx.doi.org/10.1016/j.ultras.2006.05.059

$23 \mathrm{Na}$, J.K., Blackshire, J.L. Interaction of Rayleigh surface waves with a tightly closed fatigue crack, NDT\&E International, 43 (5), 432-439, (2010). https://dx.doi.org/10.1016/j.ndteint.2010.04.003

24 Jian, X., Fan, Y., Edwards, R.S., Dixon, S. Surfacebreaking crack gauging with the use of laser-generated Rayleigh waves, Journal of Applied Physics, 100 (6), 064907, (2006). https://dx.doi.org/10.1063/1.2353892

25 Hassan, W., Veronesi, W. Finite element analysis of Rayleigh wave interaction with finite-size, surfacebreaking cracks, Ultrasonics, 41 (1), 41-52, (2003). https://dx.doi.org/10.1016/s0041-624x(02)00393-1

26 Jian, X., Dixon, S., Baillie, I., Edwards, R., Morrison, J. Integrity evaluation of steel products using EMATs, Journal of Physics D: Applied Physics, 40 (2), 300-304, (2007). https://dx.doi.org/10.1088/0022-3727/40/2/002

27 Kang, L., Dixon, S., Wang, K., Dai, J. Enhancement of signal amplitude of surface wave EMATs based on 3-D simulation analysis and orthogonal test method, NDT\&E International, 59, 11-17, (2013). https://dx.doi.org/10.1016/j.ndteint.2013.05.003

28 Parekh, J.P., Tuan, H.S. Reflection and bulk-wave conversion of Rayleigh wave at a singlenshallow groove, Journal of Applied Physics, 48 (3), 994-1003, (1977). https://dx.doi.org/10.1063/1.323721

29 Saffari, N., Bond, L.J. Body to Rayleigh wave mode-conversion at steps and slots, Journal of Nondestructive Evaluation, 6 (1), 1-22, (1987). https://dx.doi.org/10.1007/bf00566693 